\title{
Scale-dependence of seismic energy-to-moment ratio for strike- slip earthquakes in Japan
}

\author{
Yasuo Izutani ${ }^{1}$ and Hiroo Kanamori \\ Seismological Laboratory, California Institute of Technology, Pasadena, CA 91125
}

\begin{abstract}
We analyzed four pairs of a large $\left(M_{w} \approx 6\right)$ and a small $\left(M_{w} \approx 3.5\right.$ to 4$)$ shallow strike-slip earthquakes to investigate the scale-dependence of the seismic energy-tomoment ratio, an important macroscopic parameter which reflects the basic physical process of seismic slip. These earthquakes occurred in the south-western part of Japan, and high-quality close-in records (epicentral distance $<50 \mathrm{~km}$ ) are available for both the small and large earthquakes. The paired events have almost the same focal mechanism and hypocenter location. We used the spectral ratio of the paired events in order to remove the effects of attenuation along the wave propagation path and the station site response. We then estimated the seismic energy from the source spectra estimated from the spectral ratio. The energy-to-moment ratio increases with the earthquake size. This scale-dependence is very similar to that found earlier for earthquakes in Southern California.
\end{abstract}

\section{Introduction}

The seismic moment, $M_{0}$, and the radiated seismic energy, $E_{R}$, are among the most fundamental macroscopic parameters for understanding the physical process of earthquake source. The seismic energy reflects the dynamic characteristics of earthquake source while the seismic moment does the static ones. The ratio of seismic energy to seismic moment, $\tilde{e}=E_{R} / M_{0}$, can be interpreted as the radiated energy per unit area and per unit slip on the fault plane. This ratio, multiplied by rigidity $\mu$, has long been used in seismology as apparent stress [Aki, 1966; Wyss and Brune, 1970]. This ratio was recently used for interpretation of dynamic source processes of earthquakes [Kanamori and Heaton, 2000].

The energy-to-moment ratio, $\tilde{e}=E_{R} / M_{0}$, estimated for earthquakes in Southern California increases with the earthquake size [Thatcher and Hanks, 1973; Kanamori et al., 1993; Mayeda and Walter, 1994; Abercrombie, 1995]. Similar scale-dependence in energy-to-moment ratio was also found for earthquakes in the eastern Mediterranean region [Hofstetter and Shapira, 2000] and in Japan [Kobayashi et al., 2000]. The scale-dependence suggests that the dynamic source process is different between small and large earthquakes.

In practice, correction for attenuation along the path poses a severe difficulty in estimating seismic energy accurately, especially for small earthquakes. In order to overcome this

\footnotetext{
${ }^{1}$ On leave from Department of Architecture and Civil Engineering, Shinshu University, Nagano, Japan

Copyright 2001 by the American Geophysical Union.

Paper number 2001GL013402.

0094-8276/01/2001GL013402\$05.00
}

difficulty Venkataraman et al. [2001] estimated the seismic energy for the 1999 Hector Mine, California, earthquake using its foreshock and aftershock records as empirical Green's functions (EGF). They removed the effects of attenuation along the path and local site condition at regional stations by taking the spectral ratio of the main shock record to EGF. The estimate using EGF is probably more accurate than those estimated with other methods.

Recently Ide and Beroza [2001] pointed out that the seismic energy currently estimated for small earthquakes tend to be underestimated because of the limited frequency band of instruments and unreliable corrections for attenuation along the wave propagation path. They evaluated the missed energy using the $\omega^{2}$ source spectral model and concluded that the energy to moment ratio is essentially constant over a 17 orders of magnitude range of seismic moment.

Thus, whether the energy-to-moment ratio is scale dependent or not is still in question because of the large scatter in the available data set. It is important to accumulate more accurate measurements to address this question. To this end, we used the high-quality close-in records which have only recently become available in Japan to accurately estimate seismic energy for 4 earthquakes in Japan; we specifically investigate whether the energy-to-moment ratio is scaledependent or not.

\section{Data and Analysis}

We analyzed four pairs of a large and a small shallow strike-slip earthquakes which occurred in the south-western part of Japan. Table 1 lists all the earthquakes studied. The source mechanisms and the seismic moments of these events have been determined by the National Research Institute for Earthquake Science and Disaster Prevention, Japan (NIED) [Fukuyama et al., 2000, 2001]. The epicenters of the

Table 1. List of earthquakes

\begin{tabular}{ccccccc}
\hline Event & Date & $M_{w}$ & $\begin{array}{c}\text { Strike } \\
(\mathrm{deg})\end{array}$ & $\begin{array}{c}\text { Dip } \\
(\mathrm{deg})\end{array}$ & $\begin{array}{c}\text { Rake } \\
(\mathrm{deg})\end{array}$ & $N$ \\
\hline $\mathrm{A}_{1}$ & 19970326 & 6.1 & 103 & 88 & -9 & 10 \\
$\mathrm{~A}_{2}$ & 19970601 & 3.7 & 290 & 90 & 7 & 10 \\
$\mathrm{~B}_{1}$ & 19970513 & 6.0 & 101 & 85 & -2 & 8 \\
$\mathrm{~B}_{2}$ & 19970611 & 3.6 & 281 & 82 & 11 & 8 \\
$\mathrm{C}_{1}$ & 19970625 & 5.8 & 319 & 89 & 14 & 6 \\
$\mathrm{C}_{2}$ & 19970626 & 3.9 & 139 & 71 & -15 & 6 \\
$\mathrm{D}_{1}$ & 20001006 & 6.6 & 150 & 85 & -9 & 16 \\
$\mathrm{D}_{2}$ & 20001006 & 3.9 & 159 & 87 & 10 & 16 \\
\hline \multicolumn{7}{c}{$M_{w}$ and focal mechanism solutions are evaluated by National } \\
Research Institute for Earth Science and Disaster Prevention, \\
Japan. $N$ is number of records.
\end{tabular}




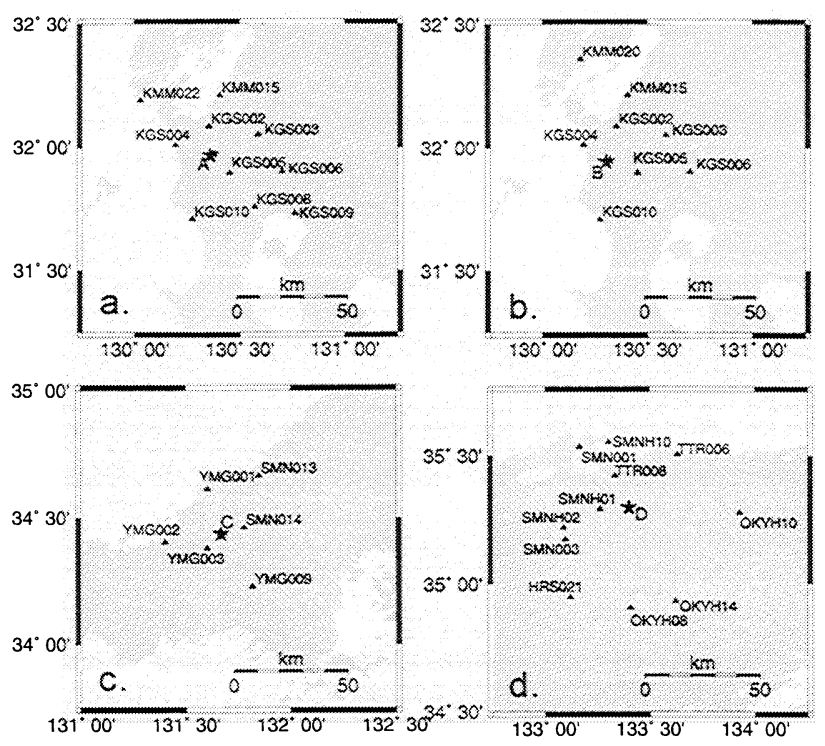

Figure 1. Locations of epicenters and stations used shown for the four pairs of a large event and a small event listed in Table 1.

earthquakes and the stations used are shown in Figure 1. The large events are strike-slip with $M_{w}=5.8$ to 6.6 and the small events are also strike-slip with $M_{w}=3.6$ to 3.9. The paired events have almost the same focal mechanism and hypocenter location. We used the spectral ratio of the paired events in order to remove the effects of attenuation along the wave propagation path and the station site response. We then estimated the seismic energy from the source spectra estimated from the spectral ratio following the method of Venkataraman et al. [2001].

We used the accelerograms recorded at K-NET and KIKNET stations operated by NIED. The epicentral distances to the stations are less than $50 \mathrm{~km}$. We used the transverse component records obtained by rotating the two horizontal components. We windowed the 70 -sec long records from the first $P$-wave arrival. Since $S$-wave is dominant on the transverse component, the Fourier spectra obtained from the records are regarded as $S$-wave spectra of the transverse component ground acceleration.

The observed ground motion spectrum is the product of the source spectrum and the transfer function for the wave propagation path. Taking the spectral ratio for each paired events, we can in effect remove the path effect and the radiation pattern effects, because the mechanisms of the paired events are very similar. Thus, the spectral ratio of the records represents the source spectral ratio of each pair. Logarithmic amplitudes of the spectral ratios at all stations are averaged for each pair to obtain the observed spectral ratio shown in Figure 2. Since the azimuthal coverage of stations around the epicenters is good, the directivity effect due to rupture propagation along the fault would be averaged out.

We assume that the source spectrum $\dot{M}(f)$ is expressed as

$$
\dot{M}(f)=\frac{M_{0}}{1+\left(f / f_{0}\right)^{n}}
$$

where $M_{0}$ is the seismic moment, $f_{0}$ is the corner frequency and $n$ is the power for the spectral decay in the frequency range above $f_{0}$. The spectral ratio for a pair of events is then expressed as

$$
\frac{\dot{M}_{1}(f)}{\dot{M}_{2}(f)}=\frac{M_{01}\left[1+\left(f / f_{02}\right)^{n}\right]}{M_{02}\left[1+\left(f / f_{01}\right)^{n}\right]}
$$

where the suffixes 1 and 2 stand for the large and small events, respectively.

The corner frequencies, $f_{01}$ and $f_{02}$, and $n$ are estimated by minimizing the sum of squared residuals between the logarithmic amplitude of the observed spectral ratio and that for the source spectral model. This method for estimating the corner frequency is the same as that used by Hough and Kanamori [2001]. Since the signal-to-noise ratio for small events at long periods is poor, the spectral ratio at frequencies lower than $0.5 \mathrm{~Hz}$ is not reliable, and the spectral ratio often falls in the low frequency range because of the increased noise for the small events, as shown in Figure 2. In the high frequency range above $5 \mathrm{~Hz}$, the observed spectral ratios for the same paired events vary considerably from station to station, probably because the path effect cannot be completely removed in this frequency range.

We used the spectral ratio over the frequency range from $0.5 \mathrm{~Hz}$ to $5 \mathrm{~Hz}$ to estimate $f_{01}, f_{02}$ and $n$ using a grid search method. The search windows are from 0.02 to $2 \mathrm{~Hz}$ for $f_{01}$, 0.2 to $20 \mathrm{~Hz}$ for $f_{02}$, and from 1.7 to 2.3 for $n$.

The observed and the best fit theoretical spectral ratios are shown in Figure 2. The optimal values for $f_{01}, f_{02}$ and $n$ are listed in Table 2.

\section{Seismic Energy-to-Moment Ratio}

Radiated energy for $S$-wave, $E_{R}$, is given by

$$
E_{S}=\frac{4 \pi}{5 \rho \beta^{5}} \int_{0}^{\infty}|f \dot{M}(f)|^{2} d f
$$

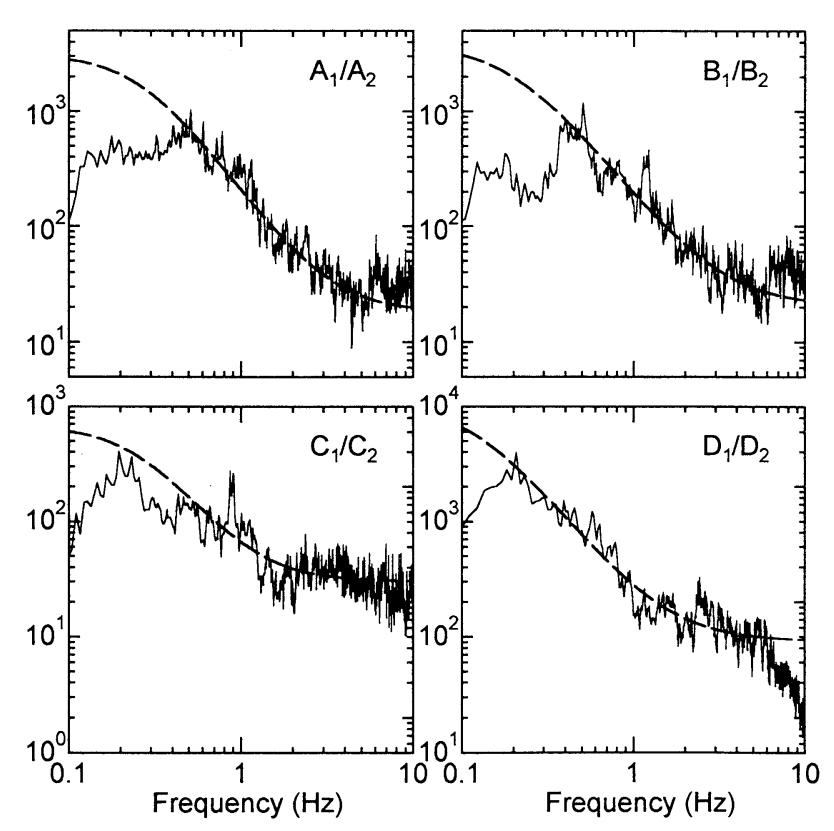

Figure 2. Source spectral ratio for the four pairs of events. The solid curves show observed spectral ratios. The dashed curves represent theoretical spectral ratios expressed by equation (2) and the parameters in Table 2. 


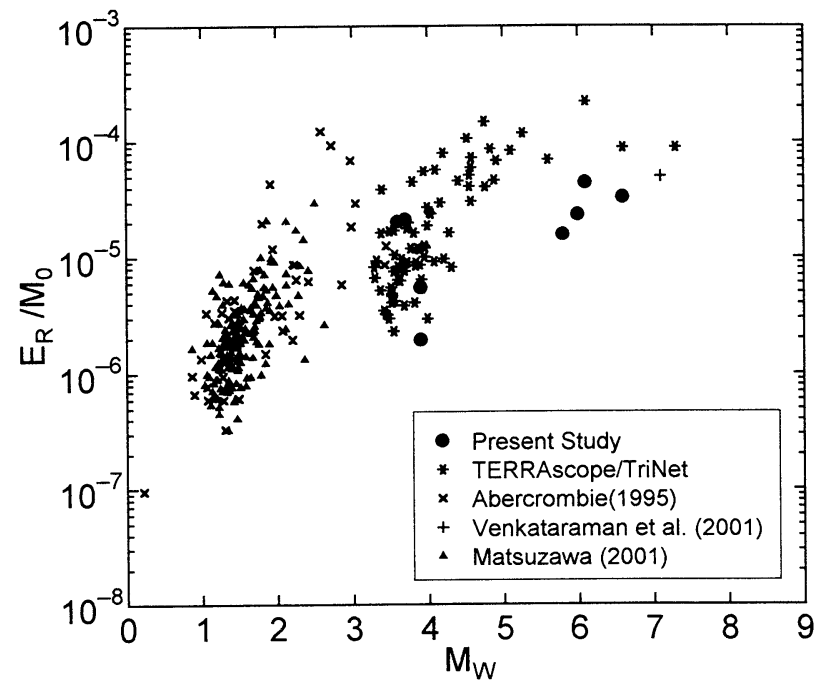

Figure 3. Energy-to-moment ratio, $E_{R} / M_{0}$, as a function of $M_{w}$. Solid circles show the results from this study. TERRAscope/TriNet indicates unpublished results obtained from the TERRAscope and TriNet data by the method of Kanamori et al. [1993]. TERRAscope/TriNet, Abercrombie [1995], and Venkataraman et al. [2001] are the results for earthquakes in Southern California. Matsuzawa [2001] is the result for shallow earthquakes in the central part of Japan.

where $\rho$ is the density and $\beta$ is the $S$-wave velocity in the source region, here assumed to be $2.7 \mathrm{~g} / \mathrm{cm}^{3}$ and $3.3 \mathrm{~km} / \mathrm{s}$, respectively [e.g., Vassiliou and Kanamori, 1982]. We assume that the source spectrum is expressed by equation (1) and used the parameters listed in Table 2. Although we assumed a particular form (equation (1)) for the source spectrum, the energy is computed by integration of the source spectrum, so that the estimate does not depend much on the particular form of the source spectrum.

Since the radiated energy for $P$-wave from a double-couple source is small compared with that for $S$-wave, it is ignored here [Haskell, 1964]. Energy estimation for the large events is more reliable than that for the small events, because the source spectra for the large events are well approximated by the theoretical ones at least in the frequency range up to $5 \mathrm{~Hz}$. The theoretical source spectra are extrapolated in the high frequency range over $5 \mathrm{~Hz}$ in estimating the seismic energy for the small events. However, since the corner frequencies are lower than $3.2 \mathrm{~Hz}$ and $n$ is about 2 (see Table2), this extrapolation would not introduce significant errors in energy estimates.

The energy-to-moment ratio, $E_{R} / M_{0}$, is between $10^{-4}$ to $10^{-5}$ for the large events, and is $10^{-4}$ to $10^{-6}$ for the small events. Figure 3 shows our results together with those from several previous studies for earthquakes in Southern California. Although the energy-to-moment ratios for the earthquakes in Japan are somewhat smaller than those for the earthquakes in Southern California, the scale-dependence is similar between Japan and California. If we combine our results with those from Matsuzawa [2001] and Matsuzawa et al. [2001] for small earthquakes in the central part of Japan, the similarity in the scale-dependence between Japan and California becomes even clear.

\section{Scale-Dependence of Energy-to-Moment Ratio}

The power for the source spectral decay in the high frequency range, $n$, in Table 2 is very close to 2 , which suggests that the source spectra for these events are well approximated by the $\omega^{2}$ source spectral model [Aki, 1967]. For the $\omega^{2}$ source spectral model,

$$
E_{R} / M_{0} \propto M_{0} f_{0}^{3}
$$

Thus, if $E_{R} / M_{0}$ is scale independent, then the commonly used scaling relation

$$
M_{0} \propto f_{0}^{-3}
$$

holds. However, if $E_{R} / M_{0}$ increases with $M_{0}$, as shown in Figure 3, this scaling relation no longer holds. For example, if $E_{R} / M_{0} \propto M_{0}^{\alpha}(\alpha>0)$, then $M_{0} \propto f_{0}^{-3 /(1-\alpha)}$. As shown in Figure 4 , the relationship between $M_{0}$ and $f_{0}$, taken from Table 2 exhibits a slope steeper than -3 , though the trend is marginally significant.

Ide and Beroza [2001] state that the energy-to-moment ratio is almost constant over 17 orders of magnitude in seismic moment. However, their conclusion relies heavily on the result by Pérez-Campos and Beroza [2001] in which the energy-to-moment ratio varies over a large range from $3 \times 10^{-4}$ to $3 \times 10^{-7}$. The large scatter may be due to inclusion of events with various fault types in various tectonic environments [Choy and Boatwright, 1995]. The energy-to-moment ratio is controlled by static and dynamic source process which may vary for different tectonic environments.

Kanamori and Heaton [2000] and Brodsky and Kanamori [2001] explained the scale-dependence of energy-to-moment ratio in terms of sudden change in friction due to microscopic mechanisms such as shear melting, fluid pressurization and elastohydrodynamic lubrication. For earthquakes with $M_{w}$ larger than 5 to 6 , dynamic friction may drop due to shear melting, fluid pressurization, and elastohydrodynamic

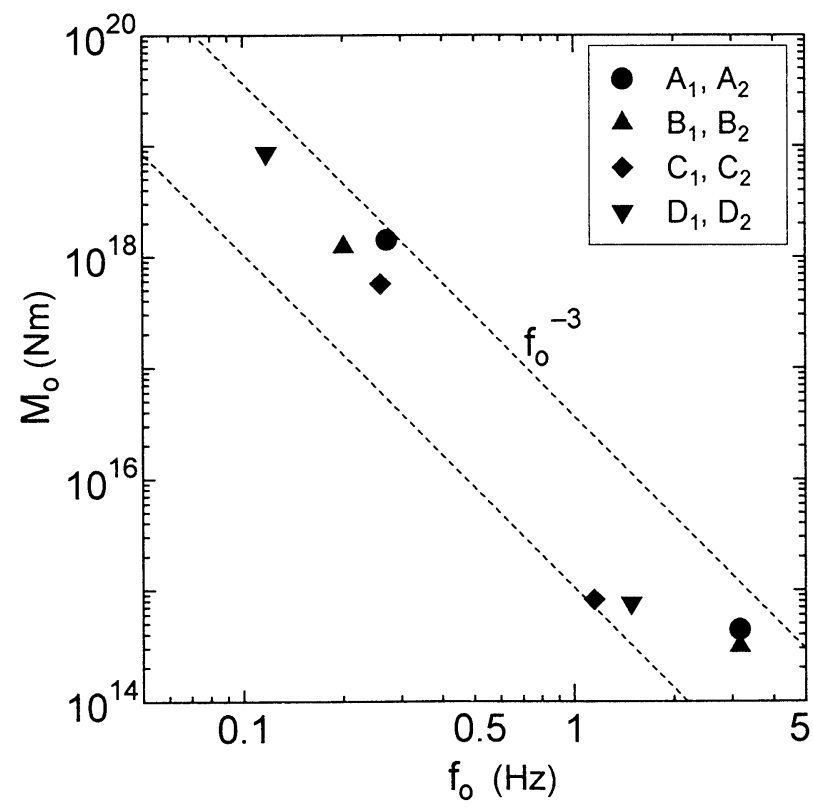

Figure 4. Relationship between seismic moment, $M_{0}$, and corner frequency, $f_{0}$. Dotted lines indicate the lines for $M_{0} \propto f_{0}^{-3}$. 
Table 2. Corner frequency and radiated energy

\begin{tabular}{lcclcc}
\hline Event & $n$ & $\begin{array}{c}f_{0} \\
(\mathrm{~Hz})\end{array}$ & $\begin{array}{c}E_{R} \\
(\mathrm{~J})\end{array}$ & $\begin{array}{c}M_{0} \\
(\mathrm{Nm})\end{array}$ & $\begin{array}{c}E_{R} / M_{0} \\
\left(\times 10^{-5}\right)\end{array}$ \\
\hline $\mathrm{A}_{1}$ & 2.1 & 0.27 & $7.3 \times 10^{13}$ & $1.40 \times 10^{18}$ & 5.2 \\
$\mathrm{~A}_{2}$ & 2.1 & 3.2 & $9.3 \times 10^{9}$ & $4.42 \times 10^{14}$ & 2.1 \\
$\mathrm{~B}_{1}$ & 1.9 & 0.20 & $3.4 \times 10^{13}$ & $1.22 \times 10^{18}$ & 2.8 \\
$\mathrm{~B}_{2}$ & 1.9 & 3.2 & $6.3 \times 10^{9}$ & $3.13 \times 10^{14}$ & 2.0 \\
$\mathrm{C}_{1}$ & 2.1 & 0.26 & $1.0 \times 10^{13}$ & $5.66 \times 10^{17}$ & 1.8 \\
$\mathrm{C}_{2}$ & 2.1 & 1.2 & $1.6 \times 10^{9}$ & $8.26 \times 10^{14}$ & 0.19 \\
$\mathrm{D}_{1}$ & 1.9 & 0.12 & $3.0 \times 10^{14}$ & $8.62 \times 10^{18}$ & 3.5 \\
$\mathrm{D}_{2}$ & 1.9 & 1.5 & $4.1 \times 10^{9}$ & $7.59 \times 10^{14}$ & 0.54 \\
\hline
\end{tabular}

$n$ is the power for the decay of the source spectrum in the frequency range higher than the corner frequency, $f_{0} . E_{R}$ is the seismic energy. $M_{0}$ is evaluated by National Research Institute for Earth Science and Disaster Prevention, Japan. $E_{R} / M_{0}$ is the energy-to-moment ratio.

lubrication and the radiated energy per unit area per unit slip becomes larger than that for smaller earthquake. Aki [2000] argues, on the basis of the size of breakdown zones and barrier intervals on a fault plane, that large $\left(M_{w}>5\right)$ and small earthquakes in California are fundamentally different. Knopoff [2000] suggests that the magnitude-frequency relationship for southern California earthquakes has two segments with different slopes, and suggests that earthquake process is scale dependent. The transition occurs at around $M_{w}=4.5$. The scale-dependence of energy-to-moment ratio in Figure 3 does not show a simple proportionality to the earthquake size. For events with $M_{w}>5$ the ratio is between $10^{-4}$ and $10^{-5}$ while the ratio for events with $M_{w}<5$ shows a larger scatter between $10^{-4}$ and $10^{-6}$. This result suggests that the rupture process of small earthquakes may be controlled by various mechanisms.

The scale-dependence of energy-to-moment ratio would provide an important clue to the difference in dynamic source process of large and small earthquakes. Also, regional variations in the scale-dependence may reflect the difference in seismogenic processes in various tectonic environments.

Acknowledgements. We thank T. A. Matsuzawa and M. Takeo who kindly sent us their unpublished data on $E_{R} / M_{0}$. Accelerograms from K-NET and KIK-NET stations operated by the National Research Institute for Earth Science and Disaster Prevention, Japan, are used in this study. Y. I. was supported by the scholarship from the Ministry of Education, Culture, Sports, Science and Technology, Japan. This research was partially supported by NSF Cooperative Agreement EAR-9909371. This is contribution 8807 of the Caltech Division of Geological and Planetary Sciences.

\section{References}

Abercrombie, R. E., Earthquake source scaling relationship from -1 to 5 using seismograms recorded at $2.5-\mathrm{km}$ depth, J. Geophys. Res. 100, 24,015-24,036, 1995.

Aki, K., Generation and propagation of $\mathrm{G}$ waves from the Niigata earthquake of June 16, 1964. Part 2. Estimation of earthquake moment, from the $\mathrm{G}$ wave spectrum, Bull. Earthquake Res. Inst. Tokyo Univ., 44, 73-88, 1966.

Aki, K., Scaling law of seismic spectrum, J. Geophys. Res.,72, 1217$1231,1967$.

Aki, K., Scale-dependence in earthquake processes and seismogenic structures, PAGEOPH, 157, 2249-2258, 2000.
Brodsky, E. E., and H. Kanamori, The Elastohydrodynamic Lubrication of Faults, J. Geophys. Res., 106, 16,357-16,374, 2001.

Choy, G. L. and J. L. Boatwright, Global patterns of radiated seismic energy and apparent stress, J. Geophys. Res.,100, 18,205-18228, 1995.

Fukuyama, E., M. Ishida, S. Horiuchi, H. Inoue, S. Hori, S. Sekiguchi, A. Kubo, H. Kawai, H. Murakami and K. Nonomura, NIED seismic moment tensor catalogue January - December, 1997, Technical Note of the National Research Institute for Earth Science and Disaster Prevention, 205, 1-35, 2000.

Fukuyama, E., M. Ishida, S. Horiuchi, H. Inoue, S. Hori, S. Sekiguchi, T. Eguchi, A. Kubo, H. Kawai, H. Murakami, S. Yamamoto and K. Nonomura, NIED moment tensor catalogue January - December, 2000, Technical Note of the National Research Institute for Earth Science and Disaster Prevention, 217, 1-131, 2001.

Haskell, N., Total energy and energy spectral density of elastic wave radiation from propagating faults, Bull. Seismol. Soc. Am., 56, 1811-1842, 1964.

Hofstetter, A. and A. Shapira, Determination of earthquake energy release in the eastern Mediterranean region, Geophys. J. Int., 143, 898-908, 2000.

Hough, S. E. and H. Kanamori, Source properties of earthquakes near the Salton Sea triggered by the 10/16/1999 M7.1 Hector Mine earthquake, Bull. Seismol. Soc. Am., submitted, 2001.

Ide, S. and G. C. Beroza, Does apparent stress vary with earthquake size?, Geophys. Res. Lett., submitted, 2001.

Kanamori, H., E. Hauksson, L. K. Hutton, and L. M. Jones, Determination of earthquake energy release and $\mathrm{M}_{\mathrm{L}}$ using TERRAscope, Bull. Seismol. Soc. Am., 83, 330-346, 1993.

Kanamori, H.. and T. H. Heaton, Microscopic and macroscopic physics of earthquakes, in GeoComplexity and Physics of Earthquakes, AGU Geophys. Mono. 120, edited by J. B. Rundle, D. L. Turcotte, and W. Klein, pp. 147-163, American Geophysical Union, Washington, D. C., 2000.

Knopoff, L., The magnitude distribution of declustered earthquakes in southern California, Proc. National Acad. Sciences, USA, 97, 11880-11884, 2000.

Kobayashi, H., J. Mori, and K. Sato, Estimates of radiated energy for moderate shallow earthquakes in Japan, $A G U$ fall meeting, S61A25, 2000.

Matsuzawa, T., Imanishi, Estimation of seismic wave energy excited by small earthquakes in the western Nagano region, Japan, Master Thesis, University of Tokyo, 2001.

Matsuzawa, T. A., M. Takeo, S. Ide, Y. Iio, and K. Imanishi, Estimation of seismic wave energy excited by small earthquakes in the western Nagano region, Japan, SSA Annual Meeting, 2001.

Mayeda, K. and W. R. Walter, Moment, energy, stress drop, and source spectra of western United States earthquakes from regional coda envelopes, J. Geophys. Res.,101, 11,195-11,208, 1996.

Pérez-Campos, X. and G. C. Beroza, An apparent mechanism dependence of radiated seismic energy, J. Geophys. Res., 106, 11,127-11,136, 2001.

Venkataraman, A., L. Rivera, and H. Kanamori, Radiated energy from the October 16, 1999 Hector Mine Earthquake: Regional and Teleseismic Estimates, Bull. Seismol. Soc. Am., submitted, 2001.

Thatcher, W..and T. Hanks, Source parameters of southern California earthquakes, J. Geophys. Res.,78, 8547-8576, 1973.

Vassiliou, M.S., and H. Kanamori, The energy release in earthquakes, Bull. Seismol. Soc. Am., 72, 371-387, 1982.

Wyss, M. and J. N. Brune, Apparent stress of earthquakes on ridges compares to apparent stresses of earthquakes in trenches, Geophys. J. R. astr. Soc., 19, 479-484, 1970.

Y. Izutani, Faculty of Engineering, Shinshu University, 4-17-1, Wakasato, Nagano, 380-8553, Japan.

(tdp0000@gipwc.shinshu-u.ac.jp)

H. Kanamori, Seismological Laboratory MC 252-21, California Institute of Technology, Pasadena, CA 91125.

(hiroo@gps.caltech.edu)

(Received May 7, 2001; accepted July 31, 2001) 